\title{
Complicated Stress-induced Cardiomyopathy and Heart Failure due to Bleeding Myoma
}

\author{
Ji-Hyoung Park ${ }^{1}$, Jaemoon Lee $^{2}$, Eunbi Lee ${ }^{3}$, Kwang Ho Lee ${ }^{4, \star}$ \\ ${ }^{1,2,3,4}$ Department of Anesthesiology and Pain Medicine, Wonju College of Medicine, Yonsei University \\ Wonju, Korea \\ ${ }^{\star}$ Corresponding author's email: khlee6006 [AT] yonsei.ac.kr
}

\begin{abstract}
Bleeding associated with uterine leiomyoma is a common complication for many women. Sustained bleeding reduces the oxygen-carrying capacity of hemoglobin. However, a hemoglobin value below 2.0 g/dL due to bleeding is not common. Such a low level compensatively increases sympathetic tone and can lead to stress-induced cardiomyopathy and congestive heart failure. We report a case of severe anemia (1.4 g/dL of hemoglobin) with acute heart failure due to myoma-related bleeding.
\end{abstract}

Keywords - Acute heart failure, Bleeding, Leiomyoma, Stress-induced cardiomyopathy.

\section{INTRODUCTION}

Bleeding associated with uterine leiomyoma is a common complication and a significant medical and social problem for many women. Although the exact pathophysiology is unknown, changes in the vascular structure of the uterus and vasoactive growth factors or receptors of the leiomyomatous myometrium have been suggested [1]. Sustained bleeding reduces the oxygen-carrying capacity of hemoglobin, resulting in a compensatory increased sympathetic tone and related increased heart rate and cardiac output. This phenomenon can lead to stress-induced cardiomyopathy and congestive heart failure [2,3]. We report a case of acute congestive heart failure in a patient with myoma-related bleeding.

\section{CASE}

A 38-year-old female $(164 \mathrm{~cm}, 54 \mathrm{~kg})$ visited the emergency department with continuous vaginal bleeding for 3 weeks and dyspnea for 3 days. Her initial vital signs were blood pressure (BP) $94 / 54 \mathrm{mmHg}$, heart rate (HR) $117 \mathrm{bpm}$, and body temperature (BT) $36.8{ }^{\circ} \mathrm{C}$. She had no medical history and no surgical history. Menarche occurred at 15 years of age, and her menstrual periods, which had usually been regular at 35 days, had been getting longer in the previous 3 months. Her vaginal bleeding required 8 to 10 medium pads each day since the last menstrual cycle. Physical examination revealed a palpable mass in the lower abdomen and blood-stained vagina. There was no sign of active bleeding in the vagina. Initial laboratory examinations revealed blood hemoglobin $(\mathrm{Hg})$ concentration of $1.4 \mathrm{~g} / \mathrm{dL}$, and brain natriuretic peptide (BNP) and Troponin I were increased to $906.88 \mathrm{pg} / \mathrm{mL}$ and $0.039 \mathrm{ng} / \mathrm{mL}$, respectively. Computed tomography (CT) of the chest showed mild bilateral pleural effusions (Fig.1). Abdominal and pelvic CT revealed a huge myoma of the uterus (Fig.2). 


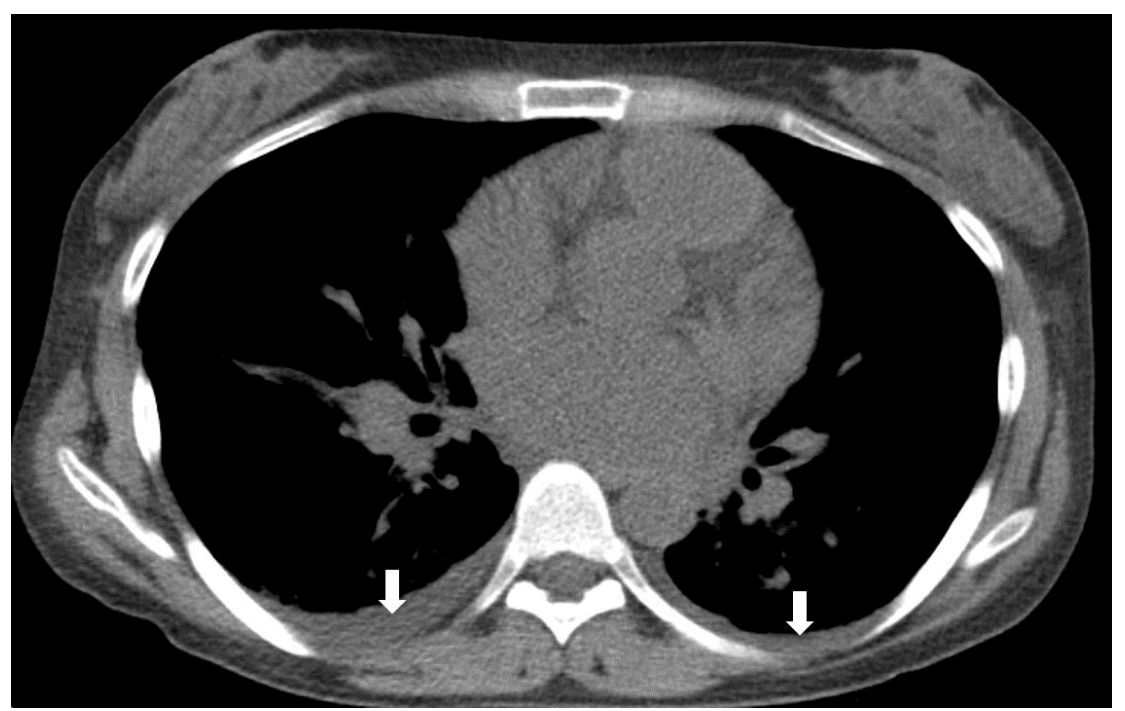

Figure 1. Chest computed tomography revealed mild bilateral pleural effusions

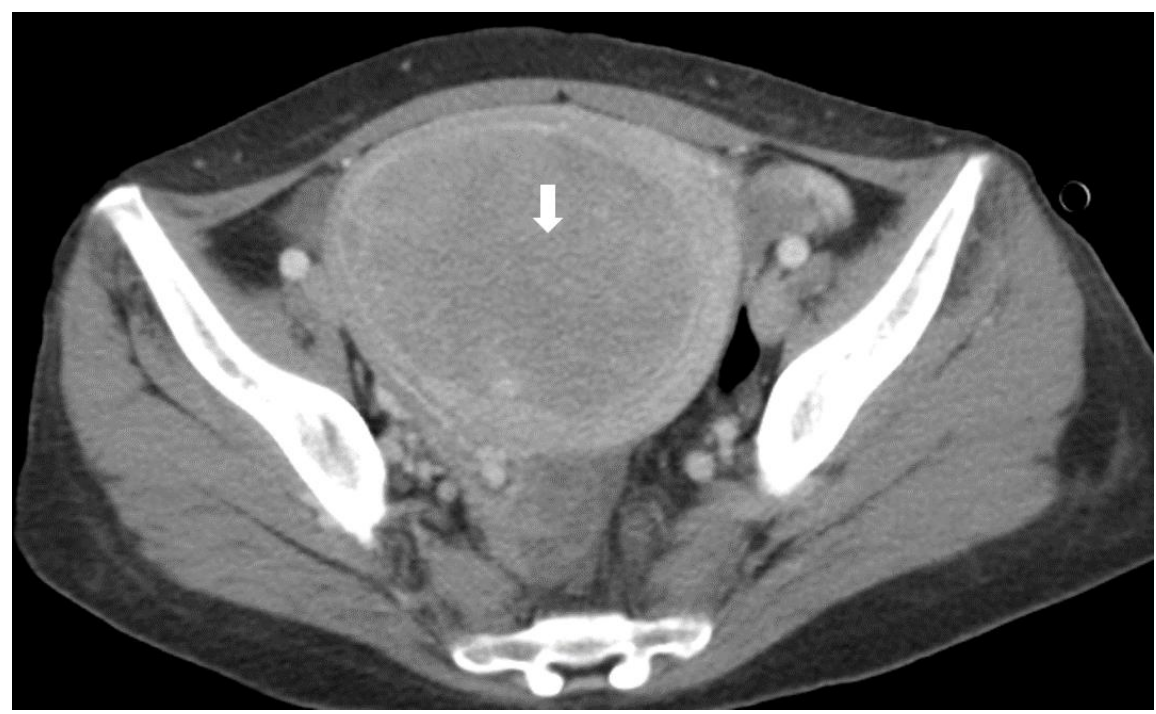

Figure 2. Abdominal and pelvic computed tomography demonstrated a huge myoma of the uterus

Echocardiography showed reduced left ventricle $(\mathrm{LV})$ systolic function (Ejection fraction $=28 \%$ ), akinesia on the anterolateral and anterior walls from the middle to apex, severe hypokinesia on the posterolateral and inferior walls from middle to apex, moderate hypokinesia on the interventricular septum from the middle to apex (Fig.3-1, 3-2), and mild pulmonary hypertension (right ventricular systolic pressure $=43 \mathrm{mmHg}$ ). For the differential diagnosis of coronary artery occlusive disease, she underwent coronary angiography that demonstrated normal coronary arteries (Fig.4). A review of the patient's examinations suggested a diagnosis of stress-induced cardiomyopathy, and her treatment comprised furosemide, spironolactone, candesartan, ivabradine, and captopril. 


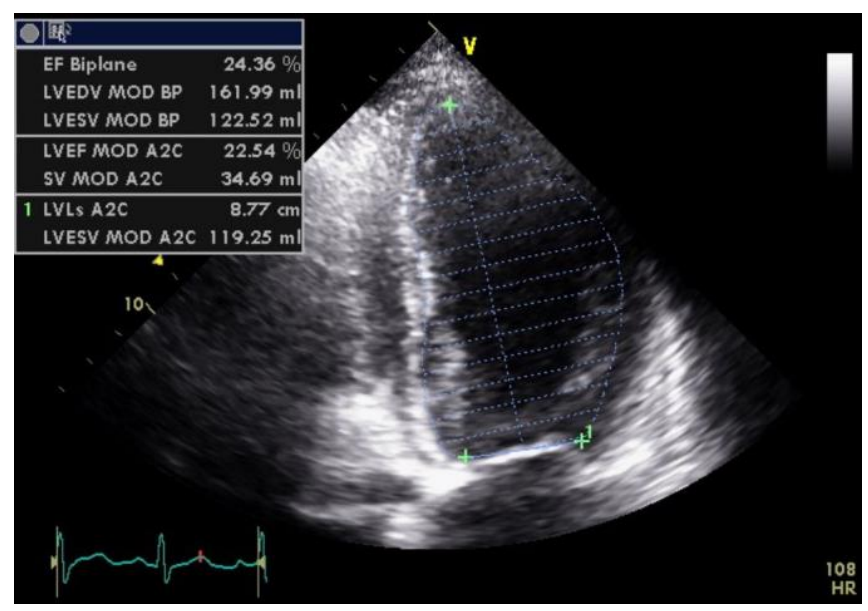

Figure 3-1. Echocardiography showed reduced left ventricle systolic function

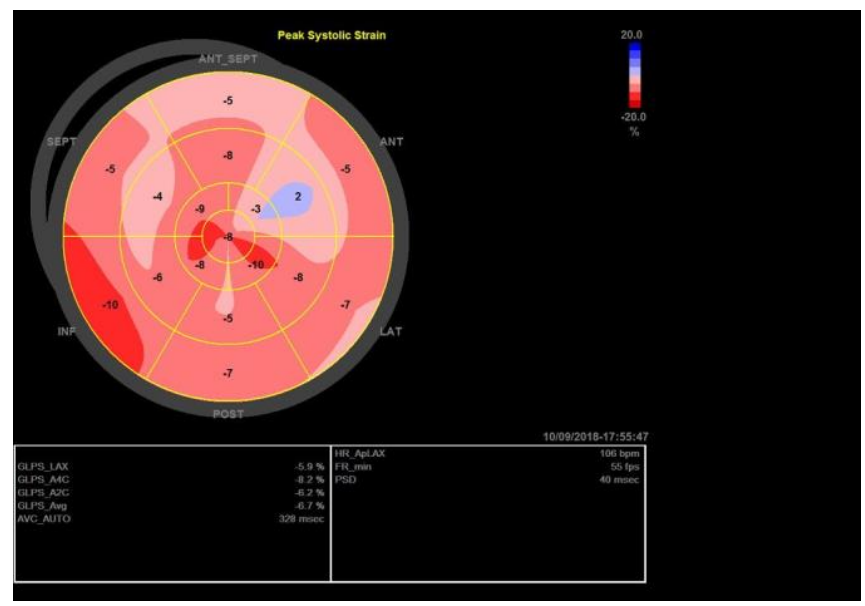

Figure 3-2. Cardiac regional wall motion abnormality
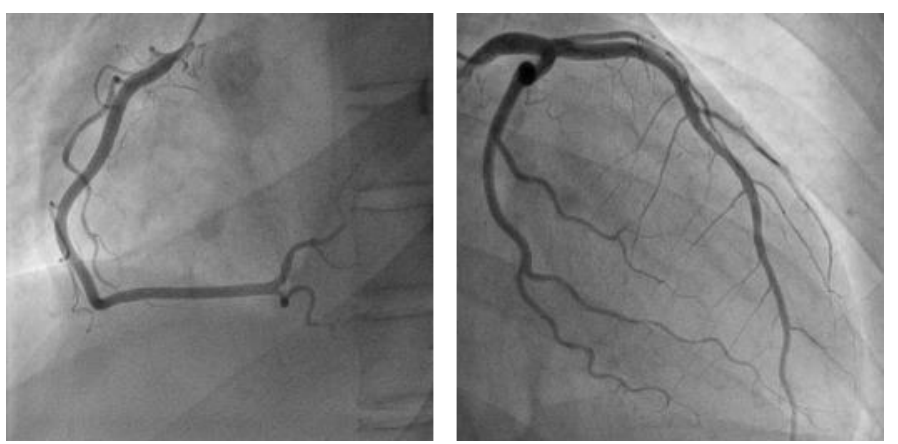

Figure 4. Coronary angiography revealed normal coronary arteries

In addition, the patient received 13 units of packed red blood cell for correction of blood hemoglobin level, and one week later, we decided to perform myomectomy for the bleeding myoma. After providing informed consent for surgery and anesthesia, the patient arrived at the operating room without premedication. Routine non-invasive monitoring was established, including electrocardiography (ECG), HR, non-invasive blood pressure (NIBP), and pulse oximetry (SpO2). Bispectral index monitoring was also performed. Her baseline HR and NIBP were $62 \mathrm{bpm}$ and 119/81 mmHg, respectively. $\mathrm{SpO} 2$ was $100 \%$ on room air, and ECG revealed normal sinus rhythm. After preoxygenation, the patient was induced into general endotracheal anesthesia with an intravenous thiopental, rocuronium, and remifentanil infusion. A modified Allen's test was normal, and an arterial line was applied to the left radial artery to monitor invasive arterial blood pressure (ABP) and pulse pressure variation continuously. In addition, Flotrac EV 1000 (Edwards Lifesciences, Irvine, USA) was added to monitor cardiac output, cardiac index (CI), and systemic vascular resistance (SVR). A central venous pressure (CVP) 
line was also applied to the patient's right internal jugular vein. Five minutes after completion of the anesthetic procedures, the surgical drapes and other preparations were placed, and surgery commenced. A 13 x 13-cm-sized myoma was excised, and 2.5 units of oxytocin were infused over 30 minutes. The duration of surgery was 55 minutes. The patient received 950 $\mathrm{mL}$ of plasma solution, and estimated blood loss was approximately $300 \mathrm{~mL}$. Intraoperatively, ABP was within $\pm 20 \%$ of baseline, CVP was 4-8 cm H2O, CI was 3.1-3.7 L/min/m², and SVR was $1629-1988 \mathrm{dynes} / \mathrm{sec}^{2} \mathrm{~cm}^{5} / \mathrm{m}^{2}$. Neither inotropes nor vasopressors were used. The patient recovered well and was discharged on the fifth postoperative day without complications.

\section{DISCUSSION}

In this case, we found that severe anemia from leiomyoma-related bleeding can cause stress-induced cardiomyopathy and acute heart failure. Uterine leiomyoma is a benign smooth muscle tumor and a common cause of abnormal uterine bleeding. About $30 \%$ of women with leiomyoma experience menorrhagia or excessively heavy menses. Changes in the venous structure of the endometrium and myometrium and overexpression of genes involved in angiogenesis and vasodilation may contribute to leiomyoma-related bleeding [1]. Treatment of leiomyoma with abnormal bleeding is roughly divided into two broad categories: surgical resection and hormone therapy. Surgical resection includes hysterectomy, myomectomy, and endoscopic resection. The second strategy is control of the leiomyoma with a steroid hormone. Although it is not optimal in all patients, surgery is the mainstay of treatment. This increases the chances of anesthesiologists caring for patients with leiomyoma in the operating room.

There is no direct literature evidence that decreased oxygen-carrying capacity due to anemia directly reduces myocardial contractility. In the present case, markers of myocardial injury (CK-MB, BNP and Troponin-I) were normal or mildly elevated, and there were no lesions on cardiovascular angiography. However, multiple regional wall motion abnormalities and severely decreased LV systolic function were observed on echocardiography.

Various forms of anemia can alter the activity of the autonomic nervous system. Low oxygen content causes the carotid body to alter the balance of the autonomic nervous system by ventilatory and cardiovascular reflexes [3]. Increased sympathetic stimulation is a major cause of stress-induced cardiomyopathy. The exact mechanism underlying the association between sympathetic stimulation and myocardial stunning is unknown. However, Ilan S. et al. noted the possibility of spasm of the epicardial coronary arteries or microvasculature. Another mechanism suggests direct myocardial injury by catecholamines [4]. Metabolic acidosis caused by hypoxia might also contribute to decreased myocardial contractility.

Treatment of congestive heart failure secondary to stress-induced cardiomyopathy remains largely empirical. Various methods may exist, but the goals of optimal anesthesia are as follows. First, maintenance of forward flow is needed to prevent coronary ischemia, pulmonary hypertension, and end organ dysfunction. The second goal is to promote inotropy without inducing or worsening ischemia, and the third is to achieve postoperative function that matches the patient's preoperative levels [5]. In the case of hemodynamic instability, it is recommended to assess left ventricular contractility by pulmonary artery catheter. Central venous pressure can indicate failure of right ventricular contraction. Transesophageal echocardiography during surgery can also monitor myocardial function in real time. Using indicators to predict fluid responsiveness and ensuring proper contractility through inotropic medicine to maintain an adequate effective circulating volume would help ensure patient survival. The patient in the present case had a serious condition, indicated by the EF reduced to $28 \%$, because of severe heart failure due to cardiac compensation with an extremely low Hg level. Therefore, bleeding myoma should be diagnosed and treated early to avoid serious complications.

\section{CONCLUSIONS}

Severe anemia caused by uterine leiomyoma may lead to stress-induced cardiomyopathy. Moreover, heart failure can occur in young patients without coronary artery disease. Therefore, in cases of persistent abnormal uterine bleeding, cardiac function should be monitored for heart failure, and consideration should be given to the use of proper inotropes for myocardial dysfunction as detected by assessment monitoring during anesthesia.

\section{REFERENCES}

1. Stewart, EA, Nowak RA., "Leiomyoma-related bleeding: A classic hypothesis updated for the molecular era", Human Reproduction Update, vol. 2, no. 4, pp.295-306, 1996.

2. Franchitto, N., Despas, F., Labrunee, M., Roncalli, J., Boveda, S., Galinier, M., Senard, J.M., Pathak, A., “ Tonic chemoreflex activation contributes to increased sympathetic nerve activity in heart failure-related anemia", Hypertension, vol. 55, no. 4, pp.1012-1017, 2010.

3. Yokusoglu, M., Nevruz, O., Baysan, O., Uzun, M., Demirkol, S., Avcu, F., Koz, C., Çetin, T., Hasimi, A., Ural, AU, Isik E., "The altered autonomic nervous system activity in iron deficiency anemia", The Tohoku J Exp Med, vol. 212, no. 4, pp. 397-402, 2007. 
4. Wittstein, IS., Thiemann, DR., Lima, JAC., Baughman, KL., Schulman, SP., Gerstenblith, G., Wu, KC., Rade, JJ., Bivalacqua, TJ., Champion, HC., "Neurohumoral features of myocardial stunning due to sudden emotional stress", N Engl J Med, vol. 352, no. 6, pp. 539-548, 2005.

5. Chua, JH., Nguyen, R., “Anesthetic management of the patient with low ejection fraction”, Am J Ther, vol. 22, no. 1, pp. 73-79, 2015. 